$81,\left.74\right|_{14}$ LA-5799-MS

12 Informal Report $\quad \begin{aligned} & \text { UC-48 } \\ & \text { Reporting Date: November } 1974\end{aligned}$

$8 n-111$

$\rightarrow$

;

\title{
Techniques for Visualizing Pion Treatment Ports at LAMPF
}

\author{
by
}

J. E. Baxnes*

R. L. Hutson

*Visiting staff member. Permanent address: Cancer Retsearch and Treatment Center, University of New Mexico, Albuquerque, NM 87106. 
In the interest of prompt distribution, this LAMS report was not edited by the Technical Information staff.

This work was supported by the Public Health Service Research Grant No. CA.14052.

Printed in the Uniled States of Amorica. Avcrilable from National Technical Information Service

.S. Department of Commerce

5285 Port Ronal Road

Price: Printed Copy \$.00 Microtiche \$2.25

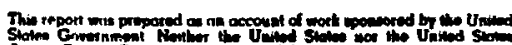

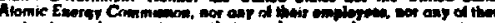

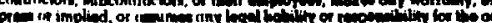

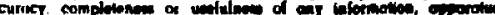

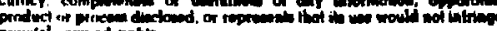

perwikity owined nok 
TECHNIQUES FOR VISUALIZING PION TREATMENT PORTS AT LAMPF ${ }^{\dagger} \neq$

by

J. E. Barnes and R. L. Hutson

ABSTRACT

\begin{abstract}
One can determine useful information about the size and stopping distribution of a therapeutic negative pion beam within a patient if one measures the spatial distribution of the beam in a plane above the patient and at right angles to the beam axis and combines the results of this measurement with previously measured divergence properties of the beam. This paper proposes a film technique for performing this determination.
\end{abstract}

\section{INTRODUCTION}

Pion irradiations of patients with cancer will require special techniques for visualizing the boundaries of the tissue volume treated. A permanent record of the treatment should be made so that tissue reactions can be correlated with dose delivered. In conventional therapy this is done with a treatment portal film, a "fast" (sensitive) film exposed to the exiting photons. The film is usually exposed twice, once with the collimator set to the field size to be treated and once with the collimator opened. This gives a record of the field to be treated superimposed on the surrounding bony anatomical features. Another film that can be made is a treatment veri,ication film. This is a very slow film exposed for the entire time of the actual treatment to make a permanent resord of what was treated.

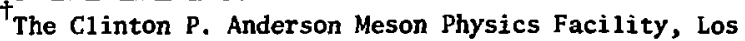
Alamos Scientific Laboratory, Los Alamos, New Mexico 87544 .

$\neq$ Th This investigation was supported by Public Health Service Research Grant No. CA-14052 from the National Cancer Institute.

*Dr. Barnes is a medical physicist with the University of New Mexico Cancer Research and Treatment Center, Albuquerque, New Mexico, and Dr. Ihtson is a nuclear physicist with the Los Alamos Scientific Laboratory, Los Alamos, New Mexico.

\section{NOTICE}

This report was prepared as an account of work sponsored by the Uniled States Government. Neither the United States nor the United States Atomic Energy Commission, nor any of their employees, nor any or their contractors, subcontractors, or their employeas, makes any warrstyty, express or implied, or assumes any logal linbility or responsibility for the accuracy, com pletentes or usefulness of any information, spparatus, product or piocoss disclosed, or represents that its use
With pions there is no exiting beam (except for contaminants) to record on film. This paper describes a method to record the anatomy of the patient in a way that can be correlated with an image of the pions recorded on filis at the entrance portal.

\section{CHARACTERISTICS OF THE PION BEAM AT LAMPF}

A vertical, almost monoenergetic pion beam will be used for radiotherapy treatments at LAMPF. The LAMPF biomedical channel consists of magnets which will be used to focus the pions at a specific depth within the volume to be treated. A plane located at this depth and perpendicular to the beam will be referred to as the focal plane. The momentum of the pions transported through the channel will be selected so that the pions stop within the volume to be treated. The stopping region within the patient will approximate a horizontally oriented cylindrical region at or near the focal plane. The depth of the stopping region will be controlled with a variablethickness energy degrader, the motion of which will rapidly move this stopping region vertically within the patient. Simultaneously, the patient will be moved slowly in a horizontal plane to distribute the stopping pions throughout the treatment volume. The treatment procedure can be considered as "fan bean" scanning. Figure 1 illustrates schematically the 
orientation of the patient with respect to the beam. Rogers ${ }^{1}$ has described the vertical scan velocity distributions required to obtain a uniform depthdose distribution with these scanning motions.

Figure 2 defines a Cartesian coordinate system for the cylindrically shaped pion stopping region. The $X$ coordinate is in the direction of the length of the cylinder. This length can be as great as 15 $\mathrm{cm}$. The near-Gaussian dose distribution in the 2 direction typically has its root-mean square (rms) value at a radius of 0.5 to $1.0 \mathrm{~cm}$. The vertical scan of the cylinder in the $Z$ direction can extend to a maximum depth of 25 to $30 \mathrm{~cm}$ in soft tissue.

Figure 3 illustrates the angular convergence of pions in the $Y-Z$ plane. The rms value for the $Y$ distribution typically occurs at a radius of 1.0 to $1.5 \mathrm{~cm}$. The difference in rms values for the $Y$ and 2 dimensions actually causes the stopping region to have the shape of an elliptical cylinder. For a typical tuning of the LAMPF biomedical channel, the pions will have an approximately Gaussian angular distribution. The rms angle of the tuning considered here is $113-m$ radians $\left(6.5^{\circ}\right)$.

Figure 4 illustrates schematically the adjacent cross sections of the stopping regions as they cross the focal plane during successive vertical scans. The horizontal scan of the patient is equivalent to a movement of the cylinder in the $Y$ direction.

The angular distribution of pions in the $x-2$ plane will be dependent on the channel tuning. Those pions which form the edges of the beam in the $x-z$ plane may be diverging, converging, or nearly parallel, depending on the tuning, Various tradeoffs of intensity, uniformity, and sharpness result from these various tunings; and an optimum configuration will be selected, based on physical measurements.

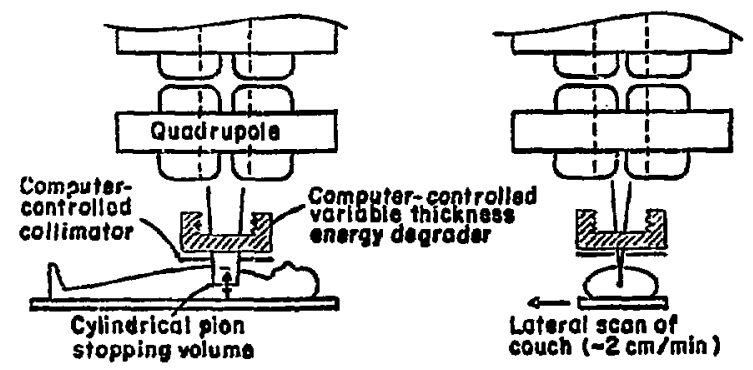

Fig. 1. Pion irradiation of patients at LAMPF biomedical facility.

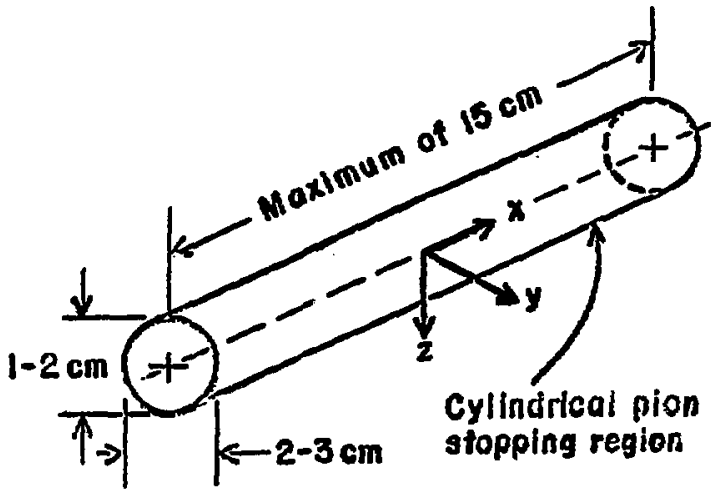

Fig. 2. Coordinate system for cylindrical pion stopping region.

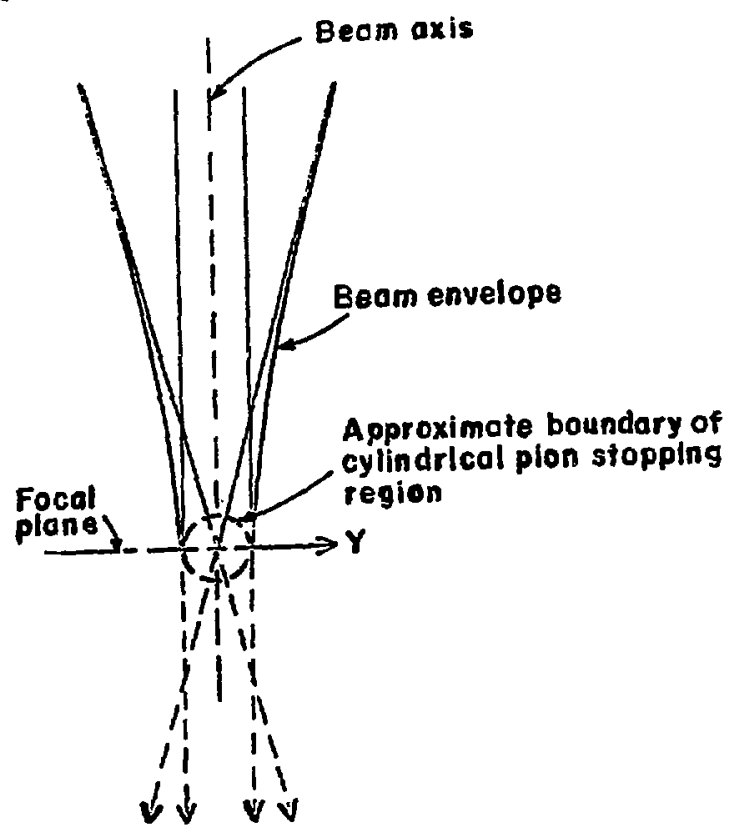

Fig. 3. Schematic representation of a cross section of the pion beam in the $Y-Z$ plane. Four representative rays are shown along with their extrapolation beyond the point where the pions stop. 


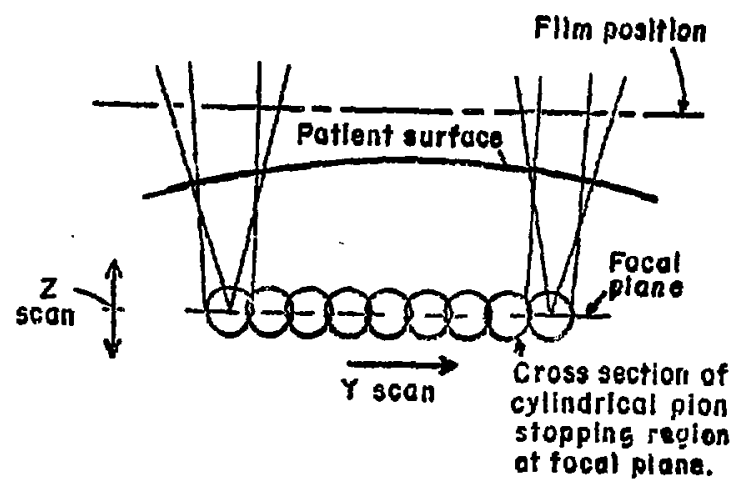

Fig. 4. Overlapping of cylindrical pion stopping regions at the focal plane.

\section{IMAGING THE PION BEAM}

Since the pions stop within the patient, it will be necessary to obtain an image of the beam above the patient (Fig. 4). If an x-ray tuoe were placed below the patient, an image of the anatomy could also be recorded on a film placed above the patient. If the two images thus obtained had equal magnifications, they could be superimposed to provide the desired correlation between beam location and anatomy.

The pion distribution is approximately Gaussian in the $\mathrm{Y}$ dimension as the beam converges at the focus. In the focal plane, and in all horizontal planes within that treatment volume, the cylindrical stopping regions will be overlapped in adjacent vertical scans so that the Gaussian distributions combine to produce a relatively uniform dose distribution. If the separation, $S$, of individual components is 1.5 times the ros value of a component, the ripple in the resultant composite distribution is less than \pm 5 percent. The distribution of pions in planes above and below the focal plane will also be Gaussian, but because of convergence above and divergence below the focal plane, the $Y$ distribution of each conponent bean will have a larger rms value in these planes.

This will result in a greater overlap of the Gaussian distributions in planes above and below the focal plane and will result in a broader edge to the bean. At the edge of a beam formed by overlapping Gaussian components, the distance from the 90 percent to the 10 percent level will be approximately 2.5 times the rms values of an individual component.

Figure 5 shows the Gaussian distributions at the focal plane for adjacent vertical scans of pions and the resulting dose profile. The distriutions are also shown in a plane above the patient. The sum of these overlapping distributions will produce an optical density on a film placed in this plane above the patient corresponding to the line shown in Fig. 5 referred to as the composite pion distribution. The composite pion distributions will have dissimilar shapes at the focal plane and the film plane; however, the $\approx 60 \%$ levels of both profiles are aligned vertically. The film image of the dose falloff near the edge of the beam is broader than the actual falloff at the focal plane. However, the film image of the anatomy in a posterior-anterior radiograph will be magnified. It is desirable to choose an optical density level in the pion beam film image such that the relationship between that level and the image of the anatomical structures is the same as the relatiunship between the "edge" (e.g., 50\% isodose level) of the pion beam at the focal plane and the anatomy.

The essential information to be obtained from this film technique is the relative positions of the beam edge and the anatomical structures at any depth of interest. Knowledge of the geometry of the $x$-ray source-patient-film configuration enables one to determine the position of an anatorical structure relative to the irradiated volume if the depth of that structure in the body is known.

In the $X-Z$ plane, the pions farming the edge of the beam may be converging, diverging, or parallel near the ends of the cylinder depending on the tuning of the channel. The width of the image of the beam recorded on a film above the patient will be dependent on this tuning. An equally magnified radiograph of the anatomy in the $x$ dimension can be made if the pions are converging. In this case the $x$-ray focal spot should be located at a distance from the pion focal plane within the patient such that the divergence angle of the $x$ rays imaging that plane is equal to the convergence angle of the pions at the edges of the beam in the $X-Z$ plane. If the pions are paral$1 \mathrm{el}$, anatomy and beam images with approximately equal magnification will result if the $x$-ray tube is placed as far away from the patient as possible and the film as close to the patient as possible. If the pions are diverging, comparable images of the pions and the anatomy can be produced only if the radiograph is taken in an anterior-posterior direction. 


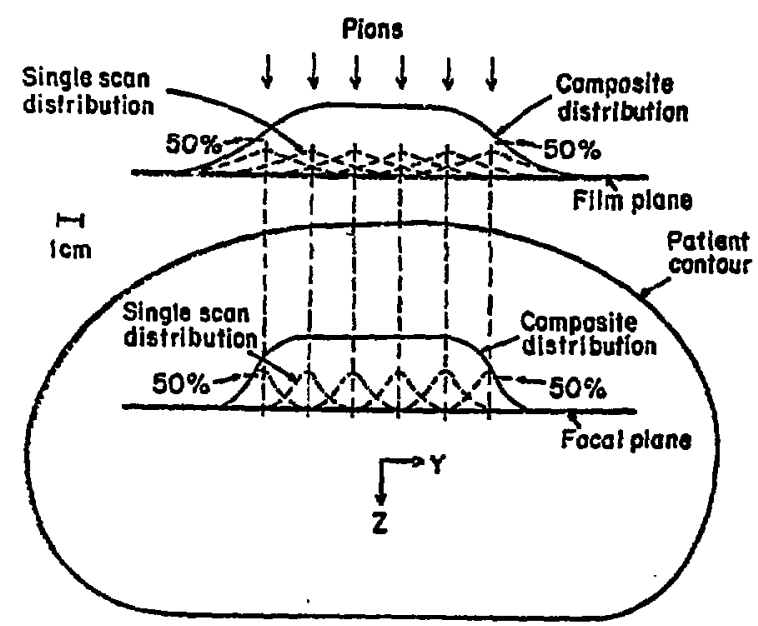

Fig. 5. Pion composite distributions at the focal and film planes from a converging pion beam scamed vertically and horizontally during irradiation.

IV. VOLUME CONSIDERATIONS

Thus far, portal films representing only a single horizontal plane through the treatment volume have been considered. However, in the patient the beam will be scanned vertically by means of a variable-thickness energy degrader placed above the patient while the focal plane of the beam is fixed at a given depth in the volums to be treated. Varying the thickness of the energy degrader results in a displacement of the stopping region of each pion along its path. The energy degrader will cause some scattering of the pions, but will not change the convergence angle significantly. Therefore, the image of the bean edges recorded on film will not change position significantly during a scan in depth. The cyitudical stopping region, however, will have a wider $Y$ listribution when the pions are stopped either above or below the focal plane than when stopped in the focal plane. If the focal plane is placed at the proximal or distal margins of the volume to be treated and the collimators are not moved during the treatment, a tapered treatment volume will be formed. If the focus is placed at mid-depth, a dual-tapered treatment volume will result, These volumes are illustrated in Fig. 6. (The shape of the sides of the dual-tapered treatment volume will be very sinilar to that obtained from two parallel opposed diverging photon beams, like those used for conventional radiation treatments.) In most cases, however, a treatmeri volume with more complex boundaries than those illustrated in Fig. 6 will be desired. This will be accomplished by varying the position of the beam collimators during the vertical scan. As the cylinder is moved either above or below the focal plane, a collimator will be moved into the edge of the beam to prevent irradiation outside the desired treatment volume. This will vary the position where the edge of the beam strikes the film and produce an unsharp image of the beam edge on the film. The greatest width in the image, however, will coincide with the widest beam longitudinal scan.

\section{v. DISCUSSION}

The techrique just described can be used for making "port" films on patients to be treated with pions at LAMPF. The beam flux can be reduced to produce an image, on a fast film placed above the patient, of the pion beam as it is planned for treatment. On the same film a magnified image of the anatomy will be made by exposure of the patient to an $x$-ray beam from below the treatment couch.
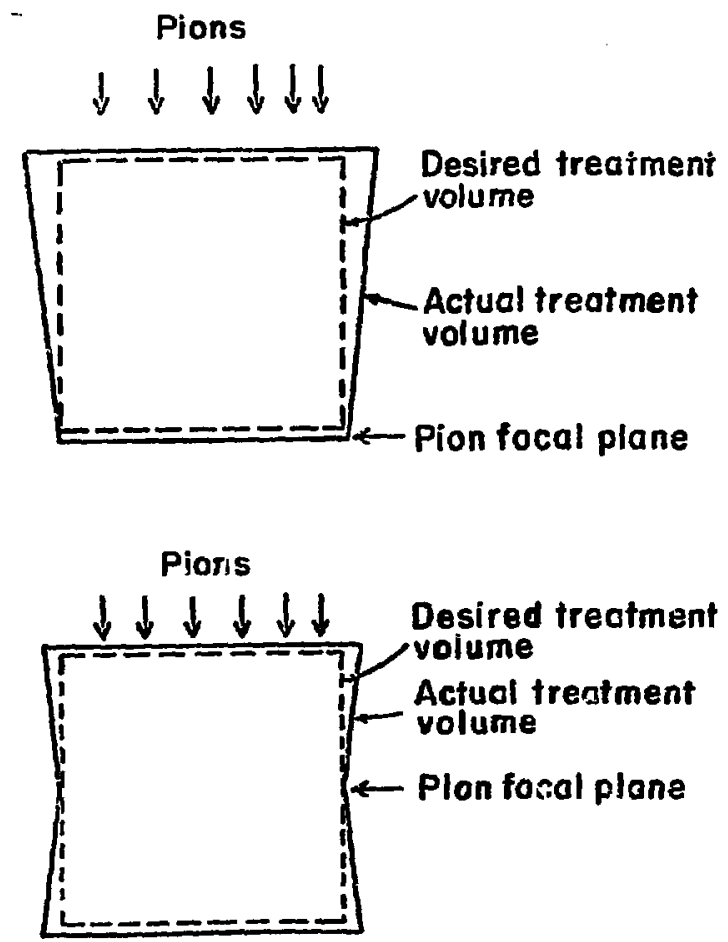

Fig. 6. Effect of placing pion focal plane at distel margin of treatment volume or at aid-depth. 
A record of the actual pion treatment can also be made if two films are used. One, a fast film to record the anatomy prior to treatment, and the other a very slow film to record the pion beam during treatment. These two films are then superimposed with the aid of alignment marks to show the port treated. This type of record will be an important aid in the analysis of the biological responses observed in the patient. It will also be extremely valuable if the treatment is interrupted for any reason before the scan is completed. It will give a basis for prescribing a supplementary field for subsequent treatment.

The technique described above for making films of pion treatment ports would complement the techniques that have been proposed for visualization of the stopping region in depth within the patient during treatment. Dean and co-workers ${ }^{2}$ have proposed the use of an Anger camera to look at the mesic $x$ rays or high-energy gamma rays emitted as the pions are captured. Sperinde et al.' 3 advocate the use of multiwire proportional counters for this purpose. Taylor et al. 4 have proposed detection of residual positron emitters for defining the pion stopping region. These techniques also face the problem of correlating the observed stopping region with the patient's anatomy. Portal films together with the image of the stopping region in depth provide information about all three dimensions of the pion treatment volume. This information will give the radiation oncologist valuable assurance that the appropriate volume of tumor-bearing tissue is being treated.

\section{ACKNOWLEDGMENTS}

The authors would like to thank Drs. E. A. Knapp and $M . M$. Kligerman for their helpful suggestions in preparing this manuscript.

\section{REFERENCES}

1. E. Rodgers, "Uniform Dose Distribution with Moving Negative Pion Beams" Los Alamos Technical Report No. LA-5326-MS, July 1973.

2. P. N. Dean and D. M. Holm, "Pion Stopping Region Visualization Experiments," Radiat. Res. 48, 201205 (1971).

3. J. Sperinde, L. E. Temple, V. Perez-Mendez, A. J. Miller, and A. Rindi, "Techniques for Mapping the Spatial Distribution of Stopping $\pi^{-}$Mesons in Tissue," Nuc1. Instrum. Meth. 97, 331-335 (1971).

4. M. C. Taylor, G. C. Phillips, and R. C. Young, "Pion Cancer Therapy: Positron Activity as an Indicator of Depth Dose," Science 169, 377 (1970). 